\title{
The Analytical Decision Model of Cleaning Space Debris
}

\author{
Jing Ding ${ }^{1, a}$ \\ ${ }^{1}$ School of Energy Power and Mechanical Engineering,North China Electric Power \\ University,Baoding 071000,China. \\ a dingjingho@foxmail.com
}

Keywords: Space debris Analytical decision model Debris cleaning up Decision problem

\begin{abstract}
In order to solve the problem B of the 2016 Mathematical Contest in Modeling(MCM) , I study the decision problem of cleaning up the space debris. To determine whether a viable commercial opportunity exits, I establish models as follows:First, I study the present situation of debris and the methods of cleaning up, among which the ground-based laser and the space-based laser technology are greatly used. In which, the method of combining the ground-based laser with the space-based laser to get the best strategies is proposed creatively.Then, I study the method of using satellite collector. According to the optimal energy and time model by using genetic algorithm we can calculate the optimal energy and time, with the practical restriction and the requirement.In addition, on the basis of the above models, I set a time-depended analytical decision model based on the empirical tractable formulas that includes the cost, benefit, and the risk factors to judge the economic feasibility of cleaning the debris qualitatively. In this model I introduce the commercial attraction coefficient $\alpha$ to assess whether the commercial opportunity exists.
\end{abstract}

\section{Introduction}

It is estimated that there are about 500,000 space debris in the earth orbit[1]. According to the NASA statistics, the number of debris will increase 3\% per year in recent time, which will bring a great threat to human space activities in the future.

Space debris is divided into three categories based on the diameter: the small space debris whose diameter is less than $1 \mathrm{~cm}$, the large space debris whose diameter is more than $10 \mathrm{~cm}$ and the dangerous space debris whose diameter is between $1 \mathrm{~cm}$ and $10 \mathrm{~cm}$ [2]. For small space debris, it can be avoided by strengthening spacecraft protection measures. As for the big space debris, because it can be monitored, spacecraft can get away from its damage by adjusting the orbit. However, the big space debris has the possibility of colliding with each other and then creates new debris, so in long-term vision the big space debris should be cleaned up. At present, the mature ways of cleaning up space debris include laser, satellite collector, powder layer, etc. This article focuses on the analysis of laser and satellite collector.

I study the principles of ground-based laser and space-based laser respectfully. I decide use laser to clean up the dangerous space debris. On the basis of which, I propose a method combining two ways of laser in order to overcome their shortcoming and find the best economic way to deal with the problem of space debris.Then I study the principle of satellite collector to clean up the large space debris.

At last, I take cost, risks and benefits factors into account by using time-dependent analytical decision model to help the government find whether the commercial opportunity exists or not and the best strategies to solve the space debris problem. And I introduce the commercial attraction coefficient $\alpha$ to determine government subsidies. I also make sensitivity analysis towards the analytical decision model.And analyse the advantages and disadvantages of the whole article.

\section{The analysis of laser and satellite collector}

Ground-based laser method. The method of ground-based laser cleaning the space debris is not directly ablating the entire debris, but parts of debris ablated to form chemical ablative. In general, the 
laser ablation will recoil impulse to debris, resulting that the debris orbit speed decreases. The smaller the orbital velocity is, the shorter the half axis length of the debris orbiting the earth. When the semi axis height decreases and the debris enters the atmosphere, the debris is entirely ablated.To remove typical debris of $800 \mathrm{~km}$ and $1500 \mathrm{~km}$,Hao Chang provided the basic information for establishing debris removing schemes and selecting suitable laser parameters[3].

Space-based laser method. The laser treatment of space debris of space-based principle is the same as the ground-based. But the space based laser is in vacuum. Therefore, using the space-based laser cleaning space debris can overcome the limitations ground-based laser. And efficiency is higher. With the development of laser technology, the future space-based laser cleaning space debris will become an efficient solution.Wulin Yang proposed three removal schemes for removing debris around typical orbits at altitudes of $1200 \mathrm{~km}, 800 \mathrm{~km}$ and $500 \mathrm{~km}$ [4].In his article,the equipments of the schemes and the technologies are compared,and it is shown that the active removal of space debris using the space-based laser system is feasible.

Combination of the ground-based and space-based laser method.Haodong Xu compared the methods of the ground-based and space-based laser as follows[5]:

\begin{tabular}{cccc}
\multicolumn{2}{c}{ Table 1 ground-based and space-based laser method comparing } \\
\hline Cleaning method & Technique difficulty & Cleaning efficiency & Cost \\
\hline Ground-based laser & Less difficulty & Low & Low \\
Space-based laser & Great difficulty & High & High \\
\hline
\end{tabular}

From the table we can see,the cleaning system of space-based laser has the technical difficulty 、 high cost disadvantages,but the removal efficiency is relatively high.In contrast,ground-baser laser cleaning system meets the principles of technical feasibility recent use and reasonable investment.According to their respective advantages and disadvantages, we can remove the space debris from high orbit to one orbit(400km for example)using the space-based laser,and remove the debris to atmosphere later by using the ground-debris.This may consume less energy than a single method.

Modeling for the method of satellite collector. For the large space debris, now we adopt the method of adjusting the spacecraft to avoid the debris. But the large space exist potential for the future space activities. So we use the satellite collector to clean up the debris. Satellite collector captures the debris in the rendezvous then brings it into the air atmosphere. When we collect one at one time, the problem is transferred into a single goal debris collection problem which is also a rendezvous problem. Alao,we can solve the problem how to complete rendezvous on multiple targets.Xiang Ye analysed this problem in detail in the article[6].In the same restriction,Xiang Ye proposed three different objective functions-optimal time、 optimal energy and optimal time and energy,and use genetic algorithm to calculate with the practicle restriction and the requirement.Government can accord to the realistic goal to adopt the objective function.

\section{The analytical decision method}

After the previous discuss the use of laser and satellite to remove space debris problem, I finally provide policy makers some advice by establishing a time-dependent model considering the costs, risks and benefits of debris cleaning up. The analytical decision model can determine whether a viable commercial opportunity exists towards the private firm.

The cost of cleaning up space debris mainly composed of two parts: one for the construction of the facilities costs or collect satellite manufacturing costs, and the other for the cost of the process equipment operation. However, due to the complexity of the cost of equipment construction and satellite launching, the costs need assessment team of experts to give an accurate estimate, so far there are not a suitable model can be used to judge the cost of these devices and their operation. However, we can estimate the cost in the angle of energy. The less energy we use, the more money we save.The decision-making process is drawn and described as follows: 


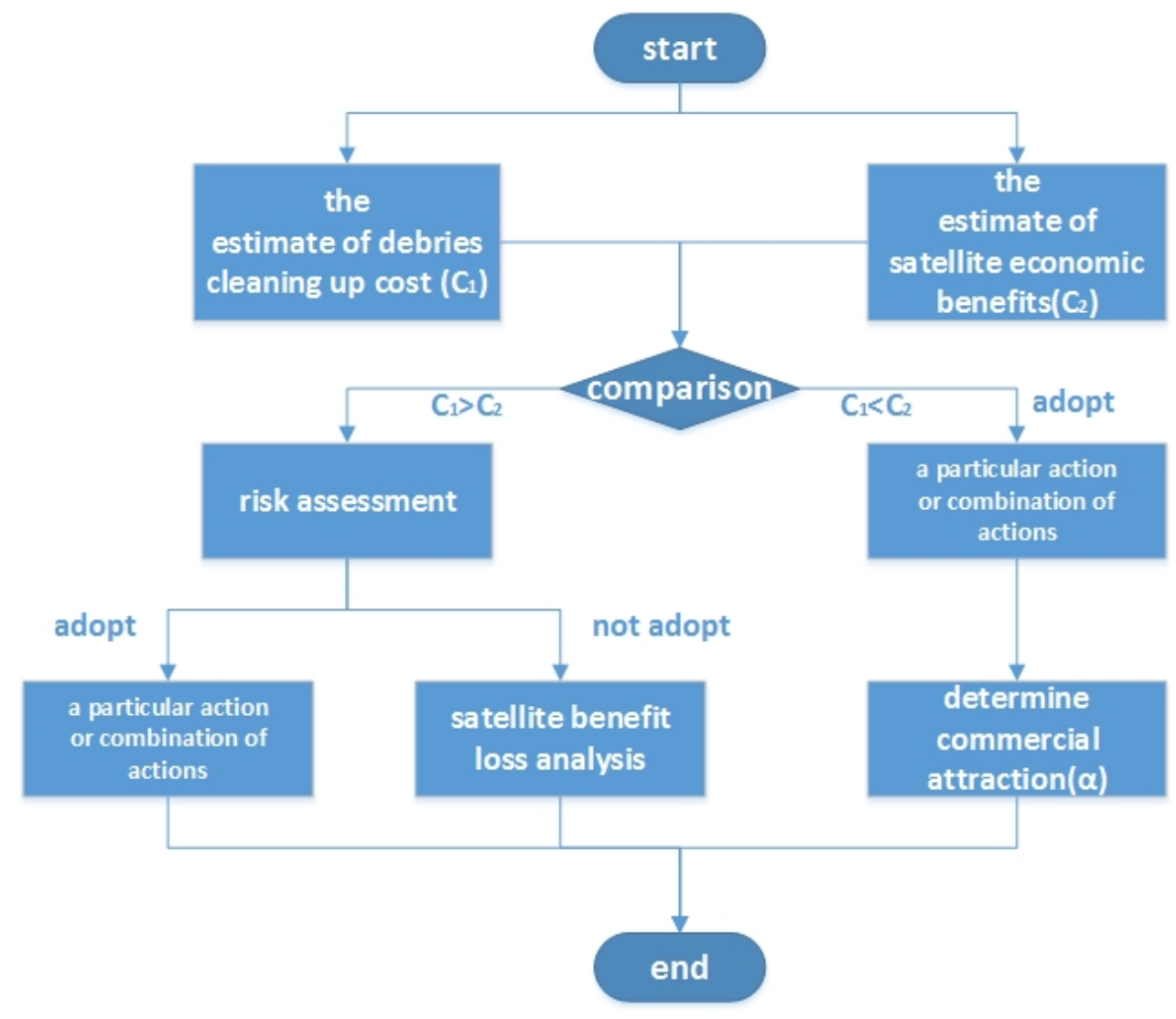

Fig. 1 decision-making diagram

Step1 The government must be clear that the type of cleaning space debris and orbital altitude to estimate the cost. This time the government should consider the most appropriate method based on the actual situation-a particular action or combination of actions to determine the lowest cost solution to the problem. To deal with low-orbit space debris whose diameter is $1-10 \mathrm{~cm}$,for example, the cost mainly composed of two parts: 1)the construction of the facilities costs and the equipment maintenance costs which need assessment team of experts to give an accurate estimate; 2)energy consumption which can use the optimal laser energy used method previously proposed to control costs reach a minimum. This time the government should have a clear expected costs, referred to as C1.

Step2 The government needs to predict the economic benefits of satellite because there may be collision which makes satellite economic decline. the formula[7]:

$$
C_{2}=\sum_{\mathrm{t}} \beta^{\mathrm{t}-1} B\left\{\left(1-Z_{\mathrm{t}}\right) V_{t}\right\}
$$

We can predict the short-term benefits C2.In the formula:

$\beta$ :the influence of the debris cleaning up policy which makes the satellite future value improve continuously.

B:the benefits that satellites can bring currently in science observations and military reconnaissance.

$\mathrm{Zt}$ : the indicator of the number of satellites falling because of the possibility of collision.

$\mathrm{Vt}$ : the number of satellites at a time.

Easy to see, if nothing is done to clean up debris policy, the benefits of the satellite will become smaller and smaller until it disappears over time.

Step3 The government compares the relationship of size between C1 and C2. 
If C1<C2:go to Step4, and a viable commercial opportunity exists. Thus the government will clean up space debris, and the government can use this project outsourced to private companies. Private companies and government all have benefits.

If C1>C2:go to Step5, and no viable commercial opportunity exists. The government has no benefit. But there is no necessary connection on government policy and benefit, the government will consider whether to clean up the debris.

Step4 Because the government's expected benefits of the satellites is higher than the debris clean up cost, the government will attract private companies through economic attractive subsidies for space debris cleaning. To measure the government's economic attractive size, I define the amount of government subsides $\mathrm{C} 3$ and commercial attraction coefficient $\alpha$,

$$
\alpha=(\mathrm{C} 2-\mathrm{C} 1) /(\mathrm{C} 2-\mathrm{C} 3) \text {. }
$$

When the amount of government subsidies exceed the costs $\mathrm{C} 1, \alpha$ is a dimensionless number greater than 1, The greater its value, indicating that government subsidies C3 is closer to satellite benefit $\mathrm{C} 2$, while private companies benefit(C3-C1) will be greater. The government may determine the value of $\alpha$ according to the actual situation to determine the economic attraction.

Step5 Under this situation, the government clean up space debris in the short term must be a loss. The government can choose to clean or not to clean up, but this time the government must consider their risks brought by options:

The government chooses to clean up space debris: go to Step6.

The government chooses not to clean up space debris: go to Step7.

Step6 Although the government has taken measures to clean up space debris currently at a loss, according to the formula, it is foreseeable that the government policy to protect the satellite will lead to decline $\mathrm{Zt}$ and rise $\beta$, so long-term economic effects of satellite accumulated should be worth the government to pay them to clean up the debris.

Step7 The government choose not to clean up space debris, there is a big risk in the future: according to Donald Kessler proposed Kessler syndrome in 1978 ,can be inferred: space debris will accumulate more and more over time, and with the collision of space debris,their quantity will continue to grow so that I can't control the development of chain reaction point. Space debris growth through

formula

to

judge:

$\mathrm{Dt}=(1+\theta) \mathrm{Dt}-1+\Gamma \mathrm{tVt}$

$\theta$ represents the growth rate of debris due to collision. The equation $(1+\theta) \mathrm{Dt}-1$ is represented by the debris growth due to collision. $\Gamma \mathrm{tVt}$ represents the debris generated by the satellite launch.

With the number of space debris increase, the debris impact on the reducing economic benefits of the satellite is becoming increasingly apparent. We can expect that satellite benefit will have enormous economic losses. Since then the economic loss to the satellite caused by space debris collisions will be much higher than original choice.

Sensitivity analysis. The sensitivity analysis is crucial to stability and rank of the reliability evaluation model. Therefore, we analyze the sensitivity of evaluation model, parameter $\beta$ is key to the evaluation of satellites economic benefits: if $\beta$ is too small, the influence of the policy is tiny; on contrary, if $\beta$ is too large, the influence is huge. In order to find out the suitable $\beta$, we change its ratio in $[0,1]$. By Normalizing the benefits: Wmax represents the maximum benefits while Wmin represents the minimum. Define :

$\alpha=$ (Wmax-Wi) / (Wmax-Wmin)

If $\alpha$ is close to 1 , that means $\beta$ is stable to the change of the possibility of collision.

Table 2 Value of $\alpha$ with the change of $\beta$

\begin{tabular}{|c|c|c|c|}
\hline 0 & & 0 & 0.8 \\
\hline .2 & .4 & .6 & \\
\hline 0 & & 0 & 0.8 \\
\hline .67 & .9 & .93 & 8 \\
\hline
\end{tabular}

From the table above, it is obvious that:

When $\beta<0.4$, economic benefits is deeply influenced by the change of the possibility of collision. 
When $\beta \geq 0.4$, economic benefits is lightly influenced by the change of the possibility of collision. As my $\beta$ is uniformly 0.4 , it means that economic benefits evaluation result is stable.

\section{Strengths and weaknesses}

Strengths.1.Analytical decision model takes cost, risks and benefits into account to analyze. It is valuable for the determination of space debris cleaning up policy.

2.Optimal energy and time model analyzes how to optimize cleaning up energy for many kinds of space debris, which has wide range of applications.

3.The performance parameters of the laser devices and other indicators are from the formal literature, so we can study on the basis of they have established .

Weaknesses.1.Analytical decision model is only able to do qualitative analysis. In addition, analytical equations are based on empirically derived, which will change under different circumstances.

2.Cost estimates for cleaning equipment can only be determined about energy consumption, however, the model cannot determine the cost of its construction and maintenance.

\section{Summary}

In the article,I study the principles of ground-based laser v space-based laser and satellite collector respectfully. I set a time-depended analytical decision model for government to clean up the space debris.According to the formal literature,we can get the minimum energy used towards different diameters and orbits. Government can estimate the cost in the angle of energy.Comparing the costs and the benefits,government can decide whether to clean up the debris.If the benefits overweigh the costs, a viable commercial opportunity exists. Thus the government will clean up space debris, and the government can use this project outsourced to private companies.On the contrary,the government is uncertain to clean up space debris.

If government does not clean up the space debris, satellite benefit will have enormous economic losses in the long term.Since then the economic loss to the satellite caused by space debris collisions will be much higher than original choice.But, it is worthy spending money on solving space debris problem for government in a long-time vision in order to attract more social forces to participate the work of cleaning up space debris even though it costs a lot at beginning, however, satellites' economic benefits in the future and space security will make up the huge expenses.

\section{References}

[1] Reference to the problem B of the 2016 Mathematical Contest in Modeling(MCM).

[2] Xianfeng Qi,Space Debris Observation Review.Aerospace China,2005,(7):24-26.

[3] Hao Chang,Xing Jin,Yanji Hong and Xiuqian Li.Modeling and Simulation on Ground-based Lasers Cleaning Space debris.Acta Aeronautica et Astronautica Sinica,2012,33(6):994-1001.

[4] Wulin Yang,Yongqiang Mou,et al.Active Removal of Space Debris by Space-based Laser System and its Feasibility Analysis.Spacecraft Environment.2015,32(4):361-365.

[5] Haodong Xu,Xiaojiang Li,Yiyong Li and Erkui Dong.Research on Technology of Space Debris Removel Using Ground-based Laser.Journal of the Academy of Equipment Command\&Technology,2011,22(3):71-75.

[6] Xiang Ye.Trajectory Design and Optimization for Space Debris Recycling. Huazhong University of Science\&Technology.2012.

[7] Molly K.Macauley.The Economics of Space Debris: Estimating the Costs and Benefits of Debris Mitigation.Acta Astronaut.2015(115):160-164. 